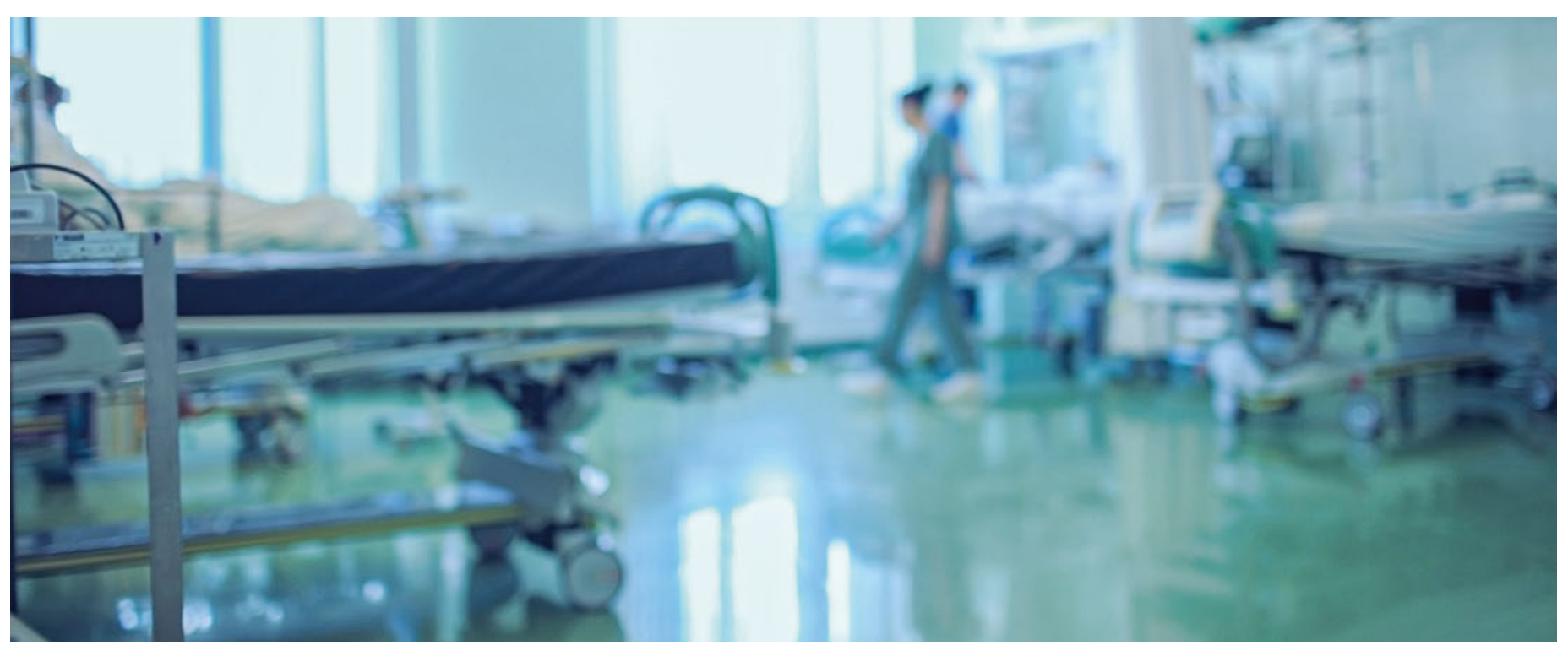

\title{
Wie umgehen mit Wirkungs- und Aussichtslosigkeit in der Medizin?
}

\section{Michelle Salathéa, Paul Hoff ${ }^{b}$}

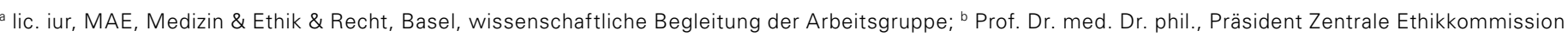
der SAMW

Wirkungs- und Aussichtslosigkeit von Behandlungen gehören zur Medizin. Oft wird die Thematik unter dem Oberbegriff der medizinischen Futility behandelt. Die Einschätzung, ob eine Therapie indiziert ist, bleibt im Einzelfall herausfordernd und es fliessen Werturteile der Fachkräfte und der Patientinnen und Patienten mit ein. Die Schweizerische Akademie der Medizinischen Wissenschaften (SAMW) hat sich mit dem Thema auseinandergesetzt und Empfehlungen veröffentlicht.

Die Beratung und Begleitung von Patientinnen, Patienten und Angehörigen in Situationen, in denen eine medizinische Behandlung wirkungs- oder aussichtslos sein könnte, ist eine zentrale Aufgabe der Medizin. Die involvierten Berufsgruppen tragen dabei eine besondere fachliche und ethische Verantwortung. Daraus resultierende Dilemmata werden deshalb auch in den medizin-ethischen Richtlinien der SAMW [1-3] ausführlich thematisiert. Dabei zeigt sich immer wieder, dass es umstritten ist, wie weit Einschätzungen der Wirkungs- oder Aussichtslosigkeit evidenz- und erfahrungsbasiert und inwieweit sie von Werturteilen geprägt sind. Dies gilt ebenso in Bezug auf die medizinische Indikation, die fachlich begründet, dass eine Behandlung geeignet und angemessen ist, um das mit der Patientin bzw. dem Patienten gemeinsam bestimmte Behandlungsziel zu erreichen.

\section{Vertiefende Empfehlungen erarbeitet}

Ausgehend von diesen Diskussionen, die während der Covid-19-Pandemie noch intensiviert wurden, hat die Zentrale Ethikkommission (ZEK) der SAMW beschlossen, vertiefende Empfehlungen zum Thema zu erarbeiten. Das Autorinnenteam wurde von einer Arbeitsgruppe der ZEK unterstützt. Im September 2021 haben die ZEK und der SAMW-Vorstand der Publikation zugestimmt. Die Empfehlungen liegen in den drei Sprachen Deutsch, Französisch und Englisch in elektronischer und gedruckter Form vor (samw.ch/empfehlungen). 
Nachfolgend werden die zentralen Aussagen zusammengefasst, ausgenommen die rechtlichen Aspekte, denen in der Publikation ein ganzes Kapitel gewidmet ist. Von den Handlungsempfehlungen zum bewussten Umgang mit dem Konzept der Futility werden beispielhaft drei ausgeführt.

\section{Das Konzept der Futility}

Wirkungslose und/oder aussichtslose Behandlungen werden oft unter dem Oberbegriff der medizinischen Futility beschrieben. Das Konzept lässt sich bis in die Zeit der Antike verfolgen, wobei es im Laufe der Zeit

\section{Es ist umstritten, wie weit die Einschätzung der Futility evidenz- und erfahrungsbasiert ist und wie weit geprägt von Werturteilen.}

hinsichtlich seiner Bedeutung und seiner Funktion einem Wandel unterlag. Die Vorstellung, dass Ärztinnen und Ärzte als Expertinnen resp. Experten über das menschliche Leben entscheiden sollten, wurde durch das Modell einer gemeinsamen Entscheidungsfindung (shared decision-making) abgelöst [4].

Das Konzept der Futility ist nicht unumstritten. Die nun publizierten Empfehlungen stellen die konzeptuellen Kontroversen dar und setzen sich mit dem Konzept der medizinischen Indikation auseinander. Dieses wird in Relation gestellt zum Konzept der Futility.
Die Einteilung in Tabelle 1 verschafft einen raschen Überblick über das Verhältnis von Wirkungs- und Aussichtslosigkeit und den Bezug zur Indikation.

\section{Medizinische Entscheidungsfindung}

Die Empfehlungen stufen das Konzept der Futility trotz aller Kritik als tauglich ein, da es auf Werten und Zielen basiert, die von vielen geteilt werden. Folglich ist es möglich, in bestimmten Konstellationen sowohl bezüglich Wirkungslosigkeit als auch bezüglich Aussichtslosigkeit von einem allgemeinen Konsens zu sprechen. Letztlich handelt es sich aber auch hier um eine Werteentscheidung, die in den Kenntnisstand der Wissenschaftsgemeinschaft eingebettet ist und gesellschaftliche Normen und Weltanschauungen widerspiegelt. Diese Evaluationen sollten möglichst transparent gemacht, reflektiert und in einen mit Patientinnen und Patienten gemeinsam gestalteten Entscheidungsprozess einbezogen werden. Dabei werden drei Kategorien unterschieden:

\section{"Medizin entscheidet»}

Bei der physiologischen Wirkungslosigkeit lässt sich empirisch feststellen (wenngleich stets eine gewisse Restunsicherheit bzw. ein Fehlerrisiko bestehen bleibt), dass eine Behandlung nicht (mehr) wirksam ist bzw. wäre. In solchen Situationen sollen medizinische Fachpersonen [5] entscheiden können, dass eine Be-

Tabelle 1: Zusammenhang von Wirkungs- und Aussichtslosigkeit mit der Indikation bei medizinischen Behandlungen.

\begin{tabular}{|c|c|c|c|}
\hline \multicolumn{4}{|l|}{ Futility } \\
\hline Wirkungslosigkeit & & Aussichtslosigkeit & \\
\hline unwirksam & wahrscheinlich unwirksam & wahrscheinlich aussichtslos & aussichtslos \\
\hline
\end{tabular}

In allen Situationen bleibt der Dialog mit den Patientinnen und Patienten bezüglich der übergeordneten und der spezifischen Therapieziele und der zur Verfügung stehenden Behandlungsoptionen zentral. Wenn die Indikation fraglich ist, sind sie in den Entscheidungsprozess einzubeziehen (shared decision-making). 
handlung nicht mehr angeboten wird (z.B. ECMO bei einer Person mit infauster Prognose). Die Entscheidung bezieht sich auf den medizinischen Sachverhalt und muss sich auf fachliche Expertise abstützen.

Es wird ein gesellschaftlicher Konsens vorausgesetzt, der den medizinischen Fachpersonen die entsprechenden Kompetenzen einräumt. Oft sind solche Situationen auf übergeordneter Ebene geregelt, etwa durch fachspezifische Richtlinien. Auch in dieser Kategorie ist der Dialog mit der Patientin resp. dem Patienten und den Angehörigen zentral. Insbesondere ist es wichtig, den Hintergrund der Entscheidung zu erklären.

\section{"Medizin berät»}

Diese Situation stellt den Regelfall dar. Medizinische Fachpersonen zeigen der betroffenen Person (oder Vertretungspersonen) die individuelle Prognose, die Behandlungsoptionen und deren Chancen und Risiken auf und schlagen eine Behandlung oder Behandlungsalternativen vor. Diese stimmt der Behandlung zu oder

\section{Stehen Wirkungs- oder Aussichtslosigkeit einer Behandlung fest, entfällt die medizinische Indikation. Dort wird es herausfordernd.}

lehnt sie ab bzw. trifft bei mehreren angebotenen Behandlungsalternativen eine Wahl. Dabei hat das Recht auf Selbstbestimmung Vorrang. Zu berücksichtigen ist, dass die Betroffenen jederzeit Behandlungen ablehnen, grundsätzlich aber nicht bestimmte Behandlungen einfordern können [6].

\section{"Grauzone»}

Therapeutische Ziele oder Massnahmen werden unterschiedlich gewichtet. In dieser Situation ist die Bewertung der empirischen Daten auch von Weltanschauung und gesellschaftlichen Werten geprägt. Soll etwa einer Patientin resp. einem Patienten das Recht auf eine Behandlung zugesprochen werden, die in den letzten 50 oder 100 Fällen wirkungslos war («Medizin entscheidet») [5-8]? Die normative Entscheidung liegt in der Festlegung der Toleranzgrenze, d.h. des cut-off (quantitative Futility) bzw. in der Bewertung der Relevanz therapeutischer Effekte (qualitative Futility).

Auch in diesen Situationen geht es darum, gemeinsam mit der betroffenen Person zu klären, ob die Behandlung gewünscht wird, obwohl die Erfolgsaussichten klein sind («Medizin berät»). Die Auswirkungen der Behandlung sind nicht nur hinsichtlich der Belastung für Patientinnen und Patienten, Angehörige oder das Behandlungsteam relevant. Bei sehr teuren Therapien gilt es auch, mögliche Opportunitätskosten zu bedenken; denn die aufgewendeten Ressourcen stehen für andere nicht mehr zur Verfügung [9]. Je nachdem ist dann eine Behandlung der Kategorie "Medizin entscheidet» zuzuordnen.

\section{Auswahl an Empfehlungen}

Stehen Wirkungs- oder Aussichtslosigkeit einer Behandlung eindeutig fest, entfällt die medizinische Indikation ("Medizin entscheidet»). Solche Situationen sind besonders herausfordernd. Medizinische Fachpersonen können sich genötigt fühlen, Behandlungen durchzuführen, obwohl diese aus ihrer Sicht nicht dem Patientenwohl dienen - beispielsweise weil Erwartungshaltungen und Druck von Patientenseite und von Angehörigen bestehen. Die ausgearbeiteten Empfehlungen - hier werden exemplarisch drei von acht wiedergegeben - geben Hilfestellung für genau solche Situationen.

1. Klärung des Behandlungsziels: Das übergeordnete Ziel der Behandlung muss mit der Patientin resp. dem Patienten bzw. dem Vertreter geklärt werden. Dabei berücksichtigen medizinische Fachpersonen Vorstellungen und Wissensstand der betroffenen Personen, tragen ihren Bedürfnissen, Wünschen und Befürchtungen Rechnung und erfragen ihre Präferenzen. Fachpersonen orientieren sich am Behandlungsziel und legen Rechenschaft darüber ab, ob das Vorgehen dem Ziel entspricht. Das Ergebnis ist in geeigneter Form zu dokumentieren.

Beispiel 1: Eine Patientin mit Gebärmutterhalskrebs entwickelt ein obstruktives Nierenversagen, das unbehandelt zum raschen Tod führen kann. Die Patientin möchte die Geburt ihres Enkelkindes in zwei Monaten erleben. Eine Dialyse kann das Leben verlängern, verändert jedoch nichts am zugrunde liegenden Tumorleiden. Sie ermöglicht es jedoch, dass die Patientin die Geburt des Enkelkindes erlebt, und kann daher sinnvoll sein.

2. Anerkennen der eigenen «Bias»: Indikationsstellungen stützen sich nicht nur auf objektive Fakten, sondern sind mitunter durch nicht-fachliche Motive beeinflusst. So können beispielsweise subjektive emotionale Bewertungen von Krankheitssituationen oder von Patientengruppen auf die fachliche Beurteilung abfärben, aber auch Angst vor heiklen Gesprächen oder Interessenkonflikte. Solche Einflüsse sind anzuerkennen und müssen kritisch reflektiert werden.

Beispiel 2: Medizinische Fachpersonen sind sich bewusst, dass sie dazu tendieren können, bei bestimmten Patientengruppen eine Therapie schneller als aussichtslos bzw. wirkungslos zu beurteilen und nicht anzubieten resp. durchzuführen, z.B. bei einer Person mit Diabetes oder einer Abhängigkeitserkrankung $[10,11]$. 
3. Betonung des Dialogischen: Die Kommunikation (das Dialogische) dient dazu, Bedürfnisse der $\mathrm{Pa}$ tientinnen und Patienten und Angehörigen zu erkennen, Vertrauen aufzubauen und gute Entscheidungen zu erreichen. Die Rolle sowohl der medizinischen Fachpersonen als auch der Betroffenen kann sich im Verlauf einer schweren Krankheit verändern. Wenn sich abzeichnet, dass die Therapien keine Wirkung entfalten, ist wichtig, den von allen Seiten akzeptierten weiteren Weg dialogisch auszuhandeln.

Beispiel 3: Bei einem schwer an Covid-19 erkrankten Intensivpatienten wird die Prognose immer schlechter. Im Gespräch mit den Angehörigen kann deren Sorge begegnet werden, der vorgeschlagene Therapieabbruch sei der Bettenknappheit geschuldet und ihr Verwandter müsse nun einem anderen Menschen Platz machen.

Die SAMW hofft, mit diesen Empfehlungen eine Reflexion über den Umgang mit Wirkungs- und Aussichtslosigkeit anzuregen, und lädt Fachgesellschaften, Pfle-

\section{Das Wichtigste in Kürze}

- Die Zentrale Ethikkommission der SAMW hat die Empfehlungen «Wirkungslosigkeit und Aussichtslosigkeit - zum Umgang mit dem Konzept der Futility in der Medizin» erarbeitet. Diese sind in drei Sprachen (Deutsch/Französisch/ Englisch) erhältlich und können kostenlos gedruckt bestellt werden unter samw.ch/empfehlungen.

- Die Einschätzung der Futility erfolgt in der Praxis durch medizinische Fachpersonen evidenz- und erfahrungsbasiert, hinein spielen aber auch Werturteile der Behandelnden sowie der Patientinnen und Patienten.

Schweizerische Akademie der Medizinischen Wissenschaften Laupenstrasse 7 CH-3001 Bern ethics[at]samw.ch georganisationen und Patientenorganisationen dazu ein, die Diskussion über diese komplexe Thematik aufzunehmen.

\section{Autorinnen der Empfehlungen}

Prof. Dr. med. Dr. phil. Nikola Biller-Andorno, Zürich; Prof. Dr. iur. Regina Aebi-Müller, Luzern; lic. iur. Michelle Salathé, MAE, Basel; Jana Sedlakova, M.A., Zürich.

\section{Mitglieder der Arbeitsgruppe der ZEK}

Prof. Dr. med. Dr. phil. Nikola Biller-Andorno, Zürich (Vorsitz); Prof. Dr. iur. Regina Aebi-Müller, Luzern; PD Dr. med. Klaus Bally, Basel; PD Dr. med. Eva Bergsträsser, Zürich; Prof. Dr. med. Dr. phil. Paul Hoff, Zürich (Präsident ZEK); Prof. Dr. med. Anja Lorch, Zürich; Dr. med. Valerie Luyckx, Zürich; Dr. med. Madeleine Mirabaud, Genf; Bianca Schaffert, MSN, Schlieren (Vizepräsidentin ZEK); Dr. rer. Medic. Ewald Schorro, Freiburg.

\section{Bildnachweis}

Sudok1| Dreamstime.com

\section{Literatur}

1 SAMW (2021). Reanimationsentscheidungen. Medizin-ethische Richtlinien. samw.ch/richtlinien

2 SAMW (2018). Umgang mit Sterben und Tod. Medizin-ethische Richtlinien. samw.ch/richtlinien

$3 \operatorname{SAMW}(2013$, aktualisiert 2020 und 2021). Intensivmedizinische Massnahmen. Medizin-ethische Richtlinien. samw.ch/richtlinien

4 Rosca A, Krones T, Biller-Andorno N. Shared decision making: patients have a right to be informed about possible treatment options and their risks and benefits. Swiss Med Wkly. 2020;150: w20268.

5 Truog RD. The Concept of Futility: Recognizing the Importance of Context. Perspect Biol Med. 2018;60(3):428-32.

6 Aebi-Müller RE. Behandlungspflichten und Behandlungsentscheide bei Ressourcenknappheit. Jusletter 1. Februar 2021.

7 Brett AS, McCullough LB. Getting Past Words: Futility and the Professional Ethics of Life-Sustaining Treatment. Perspect Biol Med. 2017;60(3):319-27.

8 Kyriakopoulos P, Fedyk M, Shamy M. Translating futility. CMAJ. 2017;189(23):E805-6.

9 Niederman MS, Berger JT. The delivery of futile care is harmful to other patients. Crit Care Med. 2010;38(10 Suppl):S518-22.

10 Lorentzon M, Nilsson AG, Johansson H, et al. Extensive undertreatment of osteoporosis in older Swedish women. Osteoporos Int. 2019;30:1297-305.

11 Bernstein SL, Yu S, Post LA, Dziura J, Rigotti NA. Undertreatment of tobacco use relative to other chronic conditions. Am J Public Health. 2013;103(8):e59-65. 